\title{
Hexagonal Photonic Crystal Fiber Behaviour as a Chromatic Dispersion Compensator of a 40 Gbps Link
}

\author{
Andrés F. Betancur-Pérez, Juan F. Botero-Cadavid, Erick Reyes-Vera, and Nelson Gómez-Cardona
}

\begin{abstract}
In this paper, the capabilities of chromatic dispersion compensation of a photonic crystal fiber with a hexagonal distribution of circular air holes was investigated. The vector finite element method with scattering boundary condition was used to analyze a set of configurations of the fiber in which the distance between air holes' centers was modified. With this method it was possible to obtain the values of chromatic dispersion and confinement factor in the $C$ fiber band. The best suited configurations were tested in a $160 \mathrm{~km}$ optical link with a bit rate of 40 Gbps. The performance was evaluated by measuring the bit error rate for a set of 20 channels with channel spacing of $100 \mathrm{GHz}$. The simulation results showed that is possible to reach values of chromatic dispersion as low as $-850 \frac{\mathrm{ps}}{\mathrm{nm} \cdot \mathrm{km}}$, confinement losses close to $10^{-3} \mathrm{~dB} / \mathrm{km}$ and good $\mathrm{BER}$ results in the order of $10^{-17}$ for a wavelength of $1550 \mathrm{~nm}$.
\end{abstract}

Keywords-BER, Chromatic dispersion, DWDM, photonic crystal fibers, vector finite element method

\section{INTRODUCTION}

$\mathbf{T}$ HE main impairments of a long-reach fiber optic transmission system with a high bitrate are the chromatic dispersion (CD) and the polarization mode dispersion (PMD). Both phenomena cause temporal broadening of the optical pulses. In the first case, this is due to wavelength dependence of the material's refractive index and the effects of refractive index difference between core and cladding of the circular waveguide (Material dispersion and waveguide dispersion). In the second case, the intrinsic birefringence induced in the optical fiber produce a temporal delay between the modes with orthogonal polarization [1]. The CD can be pre- or post-compensated, or a mixture of both [2]. This means that devices for dispersion compensation (DDCs) can be installed after a transmitter but before of the optical fiber (pre-compensation) and/or after the optical fiber but before the detector (post-compensation). DDCs can be divided in two large groups: Electronic dispersion compensators (EDCs) and Optical dispersion compensators (ODCs). EDCs use equalization techniques and digital signal processing in order to suppress the accumulated $\mathrm{CD}$ at the end of the optical link [3]-[6]. The main characteristic of EDCs is that they allow for an independent compensation for each channel on DWDM (Dense Wavelength Division Multiplexing) systems (The EDC is defined in the recommendation ITU G.959.1). ODCs, on the other hand, are based on linear and nonlinear optical phenomena. ODCs use

This work was supported by the Research Direction Office of Instituto Tecnológico Metropolitano of Medellín (ITM) through project P14102.

Andrés F. Betancur-Pérez, Erick Reyes-Vera and Nelson Gómez-Cardona are with the Electronics and Telecommunications Department, Instituto Tecnológico Metropolitano, Medellín, Colombia (e-mail: andresbetancur; erickreyes; nelsongomez@itm.edu.co).

Juan F. Botero Cadavid is with Physics School, Universidad Nacional, Medellín, Colombia (e-mail: jfbotero@unal.edu.co). special fibers with negative dispersion, some of which are known as dispersion compensating fibers (DCF) and chirped fiber Bragg grating (FBG) [7]-[9]. Since the dispersion is performed in the optical domain, there is no additional latency in the network, (unlike the ODCs, the delays are given in the electronic approach).

One of the most promising optical techniques for CD compensation is the use of photonic crystal fibers (PCF) [10][15]. These fibers consist of arrays of hollow microstructures that run parallel along the longitudinal direction of the fiber. These optical fibers can also be understood as a cylinder with multiple holes surrounding a solid core. The diameter of these holes and spacing between them are in the order of micrometers (or comparable at the wavelength of the light propagated along the fiber), hence, the light propagation is carried on through one of these two mechanisms: effective index or forbidden photonic bandgap [16]-[17]. These mechanisms of propagation, along with the radial asymmetry of the PCFs, make these fibers to show phenomena that in standard optical fibers are not possible to observe such as the large modal area, negative dispersion, or high birefringence [17]-[20].

Particularly, because the PCFs have a negative dispersion in the C- and L-band of the optical transmission systems, they can be used for CD compensation. In this article, in order to find the best setup that allows the compensation of chromatic dispersion in a 40 Gbps optical communication link, the vector finite element method (FEM) was used to make a theoretical and numerical study. Through this method, the behaviour of effective index, dispersion and dispersion slope were studied in order to obtain the required length of the dispersion compensating fiber that removes the accumulated dispersive effects. This project used the VPI Photonics software to verify the operation of the compensating fiber designed, which to our knowledge, has not been done previously. This allowed the device designed in the first stage of this work to be proven that helps to eliminate the dispersion and thus improves the quality of data received in a high-speed WDM system.

\section{METHODOLOGY}

The electromagnetic propagation modes of the microstructures can be obtained by solving the vector wave equation:

$$
\vec{\nabla} \times \varepsilon^{-1} \vec{\nabla} \times \vec{H}=k_{0}^{2} \vec{H}
$$

In equation (1), $\mathrm{H}$ is the magnetic field, $\varepsilon$ is the dielectric permittivity of the medium, $\mathrm{k} 0=2 \pi / \lambda$, is the wave vector, where $\lambda$ is the vacuum wavelength. In order to solve the wave equation, the vector finite element method with scattering boundary condition was used [21]-[22]. 
This numerical method makes possible to find the propagation effective index, $\mathrm{n}_{\mathrm{eff}}$, and the spatial distribution of the fundamental mode in a range of wavelengths from $500 \mathrm{~nm}$ to $1600 \mathrm{~nm}$. The calculated values of $n_{\text {eff }}$ are used to estimate the dispersion coefficient $\mathrm{D}[\mathrm{ps} / \mathrm{nm} \mathrm{km}]$, and the confinement loss factor $\mathrm{Lc}[\mathrm{dB} / \mathrm{km}][10]$.

$$
\begin{gathered}
D=-\frac{\lambda}{c} \frac{\partial \operatorname{Re}\left\{n_{\text {eff }}\right\}}{\partial \lambda} \\
L_{c}=\frac{2 \pi 8.686}{\lambda} \operatorname{Im}\left\{n_{\text {eff }}\right\}
\end{gathered}
$$

In equations (2) and (3), $\lambda$ is the wavelength, and $\mathrm{c}$ the vacuum light speed. The dispersion slope defined as the derivative of $\mathrm{D}$ with respect to $\lambda, \mathrm{S}=\partial \mathrm{D} / \partial \lambda$, was also calculated and can be used to estimate the coefficient $D$ of the standard single mode fiber (SSMF). In a compensated optical fiber link, it is desired that the accumulated dispersion can be approximately equal to zero.

$$
\begin{aligned}
D_{S S M F}\left[\frac{p s}{n m \cdot k m}\right] & =-\frac{S_{0}}{4}\left(\lambda-\frac{\lambda_{0}}{\lambda^{3}}\right) \\
L_{S S M F} D_{S S M F} & =L_{P C F} D_{P C F} \\
L_{S S M F} S_{S S M F} & =L_{P C F} S_{P C F}
\end{aligned}
$$

In equation (4), S_0 and $\lambda \_0$ are the dispersion slope and wavelength, respectively, when $\mathrm{D}=0$. For an appropriate compensation of the $\mathrm{CD}$, it is necessary that the lengths, $\mathrm{L}$, the dispersion coefficients, $\mathrm{D}$, and the dispersion slopes, $\mathrm{S}$, of the optical fibers, meet the conditions expressed in (5) and (6). In these equations the subscripts SSMF and PCF make reference to the standard single-mode fiber and the photonic crystal fiber, respectively. Combining equations (5) and (6), it is possible define an additional parameter known as the relative dispersion slope (RDS).

$$
R D S=\frac{S_{S S M F}}{D_{S S M F}}=\frac{-S_{P C F}}{-D_{P C F}}
$$

Figure 1 show the cross section of the PCF fiber analysed in this work. The Sellmeier equation was used to model the refractive index of the silica [23].

\section{RESULTS AND DISCUSSION}

The light propagation in PCFs is similar to that in SSMFs, meaning, both occur through the total internal reflection phenomena. However, light propagation parameters, such as the dispersion and the effective index have a strong dependence with the diameter $d_{i}$ of the holes. The design used in this paper is based on the work presented somewhere else [24]. After a large number of numerical simulations, a set of configurations with interesting chromatic dispersion curves was chosen. These configurations were designated as PCF A, PCF B, PCF C, PCF $\mathrm{D}, \mathrm{PCF} \mathrm{E}$ and PCF F. They were analyzed to observe the behavior of dispersion while the profile of the microstructure varies. Through this way it was established the most adequate

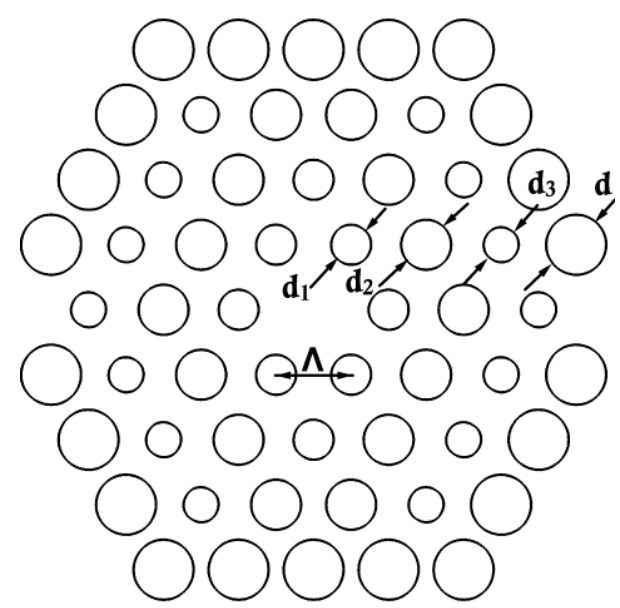

Fig. 1. Transversal section of the analysed PCF.

option to compensate $\mathrm{CD}$ in a telecommunication optical link. The PCF was simulated, keeping the microstructure period $\Lambda=0.8 \mu \mathrm{m}$ and the diameter of the holes in the fourth ring $\mathrm{d}_{4}=0.64 \mu \mathrm{m}$ constant. The diameters $\mathrm{d}_{1}, \mathrm{~d}_{2}$, and $\mathrm{d}_{3}$ corresponding to the holes' diameters in the first, second and third ring, respectively, were varied as shown in Table I.

The results acquired through the finite elements method are illustrated in Figures 2(a) and 2(b).

TABLE I

GEOMETRIC PARAMETERS OF THE ANALYSED PCFS

\begin{tabular}{ccccccc}
\hline \hline \multirow{2}{*}{ Parameter } & $\begin{array}{c}\text { PCF } \\
\text { A }\end{array}$ & $\begin{array}{c}\text { PCF } \\
\text { B }\end{array}$ & $\begin{array}{c}\text { PCF } \\
\text { C }\end{array}$ & $\begin{array}{c}\text { PCF } \\
\text { D }\end{array}$ & $\begin{array}{c}\text { PCF } \\
\text { E }\end{array}$ & $\begin{array}{c}\text { PCF } \\
\text { F }\end{array}$ \\
\hline$d_{1}[\mu \mathrm{m}]$ & 0.64 & 0.34 & 0.64 & 0.34 & 0.34 & 0.34 \\
$d_{2}[\mu \mathrm{m}]$ & 0.64 & 0.64 & 0.64 & 0.64 & 0.34 & 0.34 \\
$d_{3}[\mu \mathrm{m}]$ & 0.64 & 0.64 & 0.34 & 0.34 & 0.34 & 0.64 \\
\hline \hline
\end{tabular}
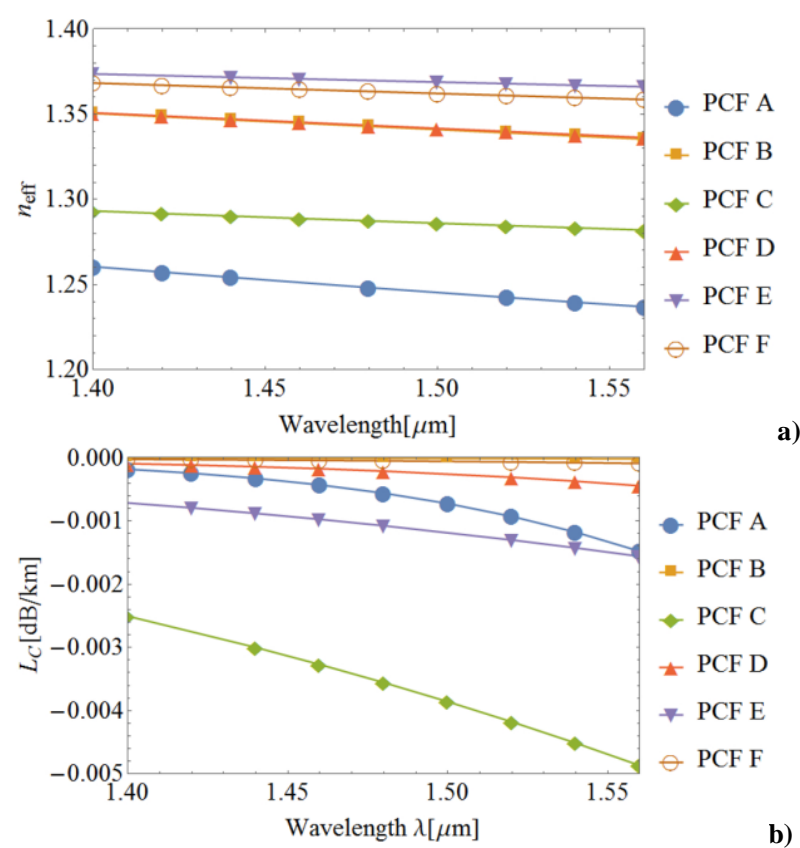

Fig. 2. (a) Effective index neff and (b) Confinement factor loss Lc for different PCF configurations. 
It can be noticed that while the wavelength increases, the value of the effective refractive index of the analyzed configurations decreases. This behavior is mainly due to the fact that smaller wavelengths of the light traveling along the fiber are confined in the center of the microstructure. For this reason, the confinement losses illustrated in Figure 2(b) show null values at lower wavelengths. Furthermore, it can be seen how the PCF C configuration shows the greatest confinement losses in the telecommunications frequency range. This behavior is a result of the variation of $d_{3}$, whose change causes a low confinement of the energy in the core of the PCF at higher wavelengths $(\mathrm{C}$ band, $1530 \mathrm{~nm}-1565 \mathrm{~nm}$ ). On the other hand, PCF B structure shows almost null losses in the band, and this can be attributed to the use of bigger holes, along with the fact that the 2nd and 3rd rings help to improve the light confinement within the core.

Using the obtained data from COMSOL for the effective index
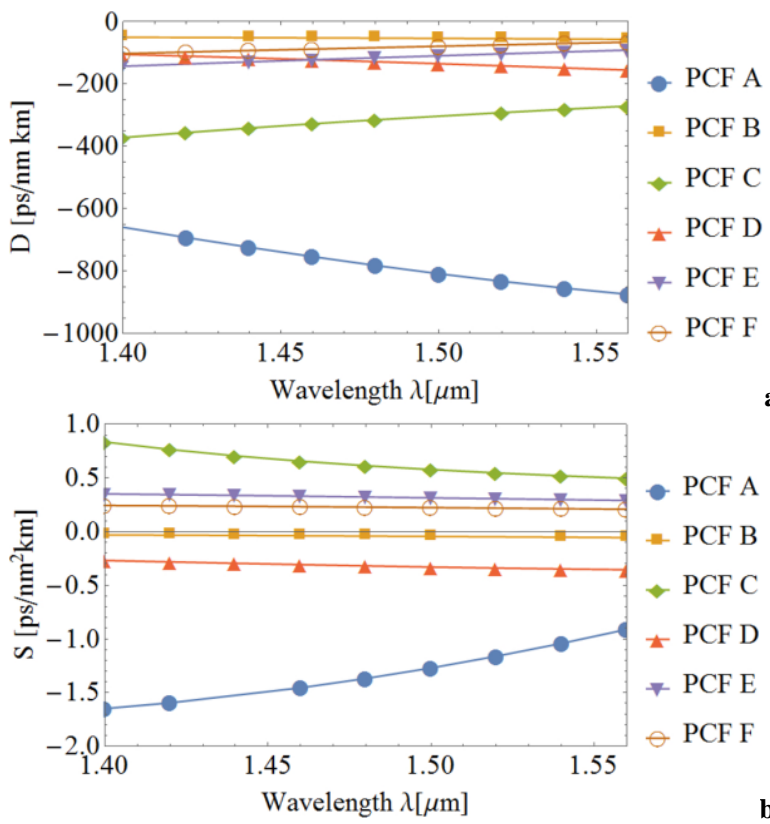

Fig. 3. (a) Dispersion D and (b) Dispersion slope $\mathrm{S}$ for different PCF configurations.

$\mathrm{n}_{\mathrm{eff}}$, the dispersion curves were calculated for every PCF configuration. As it can be seen from the obtained results in Fig. 3(a), although the configuration PCF C have negative values of dispersion (parameter $\mathrm{D}$ in the wavelength rage of the telecommunications band), Fig. 3(b) shows that the value of the dispersion slope $\mathrm{S}$, for the same wavelength range have a nearly constant positive value. Hence, these curves do not meet the imposed condition by equation (5), which requires a positive RDS. Those conditions are only met when both parameters have negative signs. On the other hand, from the findings of Fig. 3(a) and Fig. 3(b), the PCF A, PCF B, and PCF D configurations have negative values for both parameters $D$ and $S$, therefore meeting the required conditions. Thus becoming promising configurations that can possibly achieve the CD compensation required for standard SMF-28 fibers. Although, the value of the RDS must be positive, such as it is illustrated in Fig. 4, both D and $S$ parameters must be negative in order to equalize the $C D$ of the standard single mode fibers.
Table II shows, as an example, what length the proposed PCF fibers should have in order to compensate the dispersion caused in $100 \mathrm{~km}$ of standard SMF-28 fiber, as well as the maximum loss in $\mathrm{dB}$ for some wavelength values in the C-band.
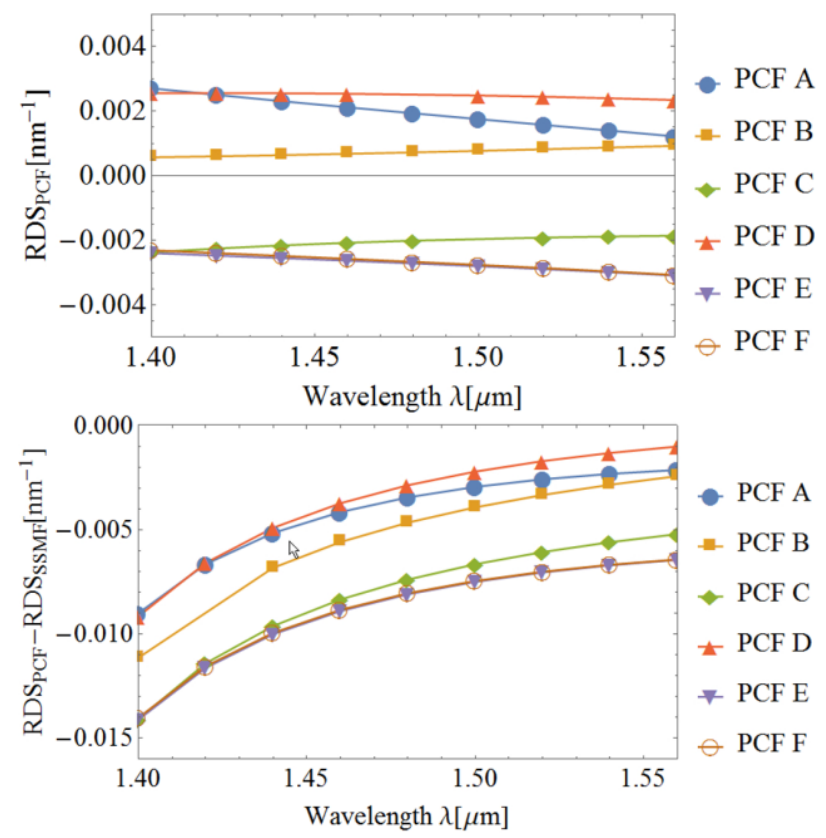

Fig. 4. RDS of the fiber after compensation using the different proposed PCF

TABLE II

LENGTH OF FIBER LPCF AND CONFINEMENT LOSS LC FOR CD COMPENSATION OF 100KM OF STANDARD SMF-28 D

\begin{tabular}{ccccc}
\hline \hline$\lambda[\boldsymbol{\mu m}]$ & $\begin{array}{c}\mathbf{L}_{\mathbf{P C F}} \mathbf{A} \\
{[\mathbf{k m}]}\end{array}$ & $\begin{array}{c}\mathbf{L}_{\mathbf{P C F}} \boldsymbol{B} \\
{[\mathbf{k m}]}\end{array}$ & $\begin{array}{c}\mathbf{L}_{\mathbf{P C F}} \boldsymbol{D} \\
{[\mathbf{k m}]}\end{array}$ & $\begin{array}{c}\boldsymbol{L}_{\boldsymbol{C}} \\
\mathbf{M a x} .[\mathbf{d B}]\end{array}$ \\
\hline 1.540 & 2.19 & 21.2 & 9.48 & $\approx 4.45 \times 10^{-3}$ \\
1.550 & 2.15 & 20.68 & 9.18 & $\approx 4.47 \times 10^{-3}$ \\
1.560 & 2.09 & 20.24 & 8.95 & $\approx 4.5 \times 10^{-3}$ \\
\hline \hline
\end{tabular}

\section{A. Performance in DWDM systems}

In order to prove the performance of the designed PCF optical fibers, the optical communications simulation platform VPI Transmission maker was used. With the purpose to simulate the designed PCF, a module with the dispersion curves was created on VPI. The dispersion curves of the PCFs designated as PCF A, PCF B, and PCF D were tested. These configurations were chosen due to the negative nature of both, the dispersion slope and CD parameter, as previously shown. Furthermore, the magnitude of the dispersion slopes $\mathrm{S}$ for these configurations showed to be relatively low.

For the simulation, the setup illustrated in Fig. 5 was used, which can be described as a point-to-point DWDM link with $160 \mathrm{~km}$ in length, $40 \mathrm{Gbps}$ bit rate, a booster next to the transmitter, and a preamplifier next to the receiver with gains of $13 \mathrm{~dB}$ and $16 \mathrm{~dB}$, respectively, and a $5 \mathrm{~dB}$ noise figure.

The standard single mode fiber (SSMF) distorts the signals by the linear effect of the $\mathrm{CD}$. This parameter is normally positive in the C-band with a magnitude of $16[\mathrm{ps} /(\mathrm{nm} \cdot \mathrm{km})]$ at a 


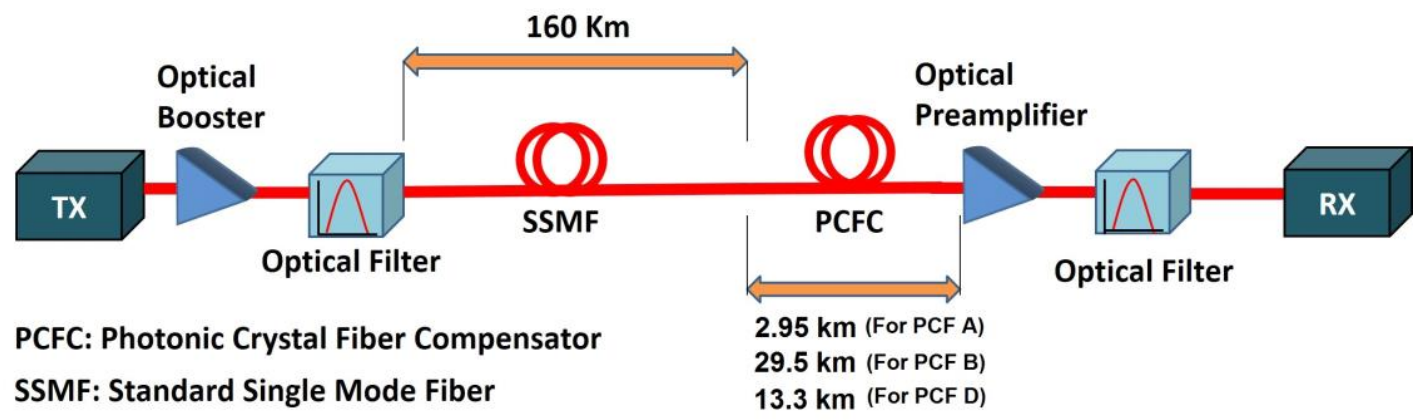

Fig. 5. Simulation scheme of the point-to-point DWDM link

frequency of $193.1 \mathrm{THz}$. This dispersion tends to accumulate as the length of the optical fiber becomes larger. As previously mentioned, to mitigate this phenomenon, $\mathrm{CD}$ compensation is employed by inducing a negative dispersion so that the accumulated dispersion is close to zero or is within a tolerance margin that the receiver can withstand. Consequently, the dispersion compensator must have a dispersion slope with an opposite sign to that one of the SSMF fiber.

In the simulation setup, data acquired in the design of the PCFs was used. Other parameters used in the simulations were: a continuous wave laser with a frequency of $193.1 \mathrm{THz}$ with a linewidth of $800 \mathrm{MHz}$, and $1 \mathrm{~mW}$ power. The laser was modulated at a $40 \mathrm{Gbps}$ bit rate with a Mach-Zehnder modulator with a $35 \mathrm{~dB}$ extinction ratio, voltage of the RF signal and $\mathrm{V} \pi$ equal to $1 \mathrm{~V}$. The insertion loss was equal to $6 \mathrm{~dB}$ and the pulse format was NRZ (Non-Return-to-Zero). The parameters of the optical fiber were those of a SSMF, whose specifications can be found in the ITU-T G.652. The propagation of the waves was simulated with the split step Fourier transform. The dispersion compensator module used the data acquired from COMSOL. In order to limit the action of the ASE (Amplified Spontaneous Emission) noise, optical filters were used with a first-order Gaussian transference function where the central frequency corresponded to the operation wavelength, and the FWHM (Full-Width-at-Half-Max) bandwidth was equal to $160 \mathrm{GHz}$.

Fig. 6 shows the captured signal at the end of a noncompensated optical fiber link, where clearly the distortion of the shape of the pulses can be noticed. This as an effect of the CD from the SSMF.

As detectors, PIN photodetectors with dark current of $2 \mathrm{nA}$, a responsivity of $1 \mathrm{~A} / \mathrm{W}$, and $1 \mathrm{pA} / \mathrm{Hz}$ of thermal noise current spectral density were used. A fourth-order Bessel low-pass filter with $30 \mathrm{GHz}$ bandwidth at $-3 \mathrm{~dB}$ was used to mitigate the noise in the electric domain. In order to test the performance of the PCFs in different channels, 20 channels with a spacing channel of $100 \mathrm{GHz}$ among them were transmitted following the ITU spectral grid. For the PCF A, the compensated eye diagrams of the channel with the biggest $(192.9 \mathrm{THz})$ and lowest distortion (193.1 THz) are illustrated in Fig. 7.

In Fig. 7 a very similar vertical closing of the eyes can be noticed, this is due to the fact that both channels are influenced by an approximately similar attenuation. The power spectral density of the noise present in the optical amplifiers is approximately the same in both channels. However, it can be seen that the channel with a frequency of $192.9 \mathrm{THz}$ experienced a greater jitter due to the CD induced by the SSMF,
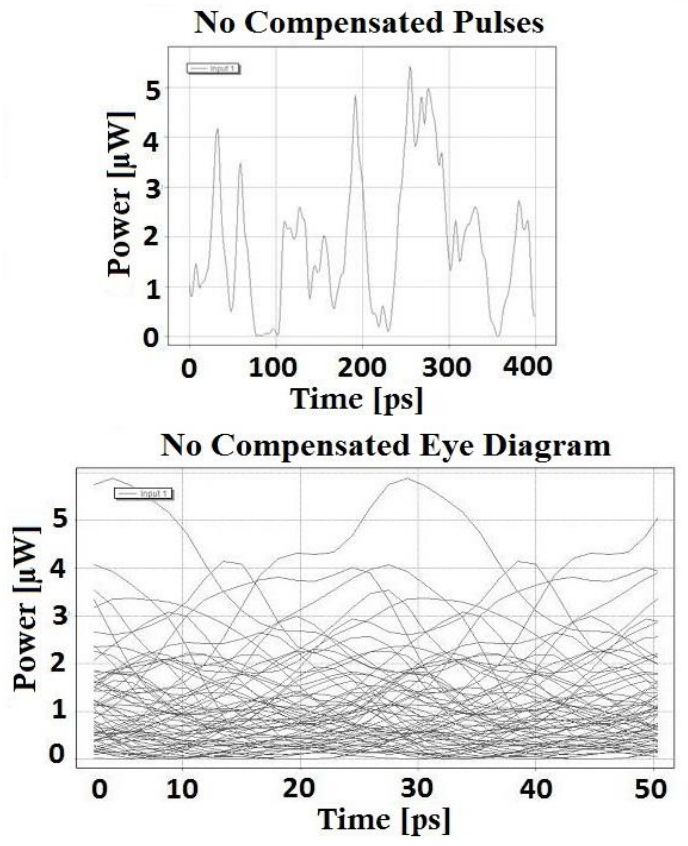

Fig. 6. No compensated pulses after $160 \mathrm{~km}$ fiber link.
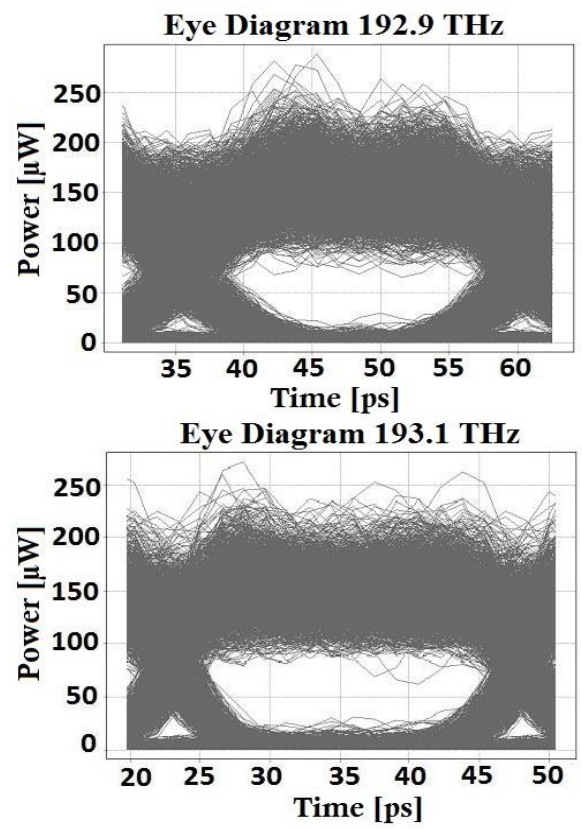

Fig. 7. Eye diagrams after using PCF A as compensator, taking as a reference a $\log (\mathrm{BER})$ equal to -12 


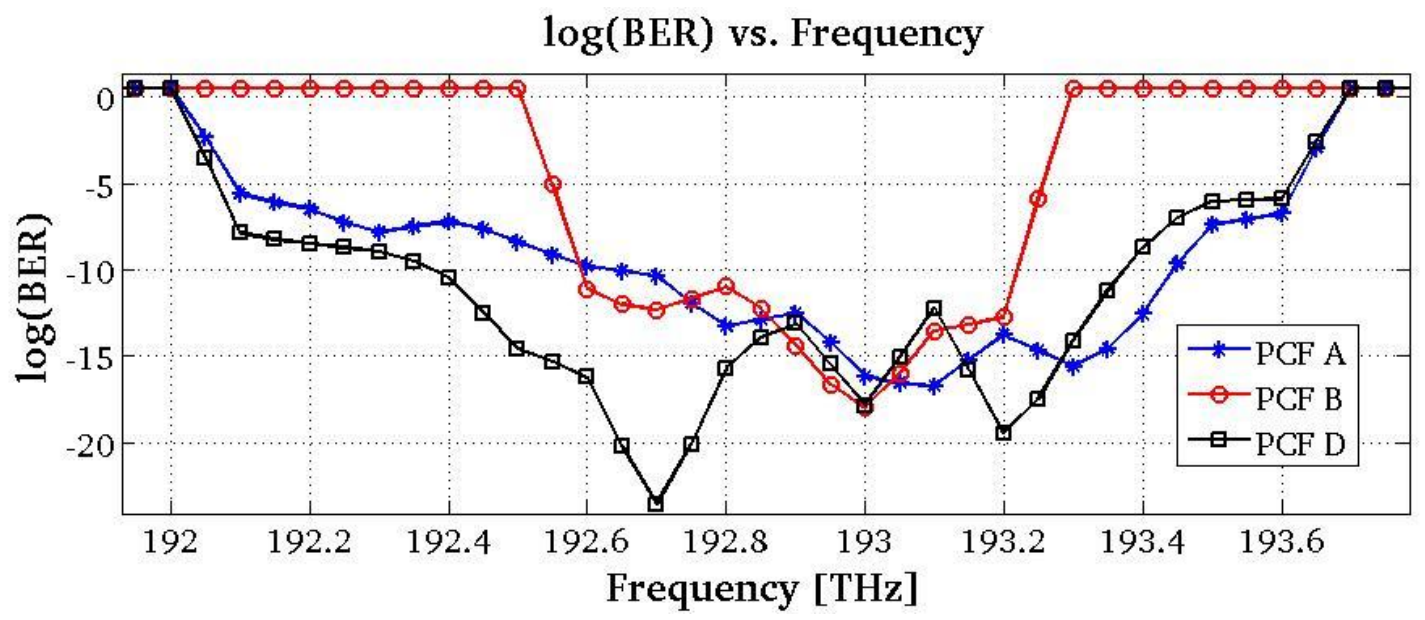

Fig. 8. BER performance after using the designed PCFs in a frequency spam of $1.7 \mathrm{THz}$

and the compensation of the PCF was less than that of the channel with a frequency of $193.1 \mathrm{THz}$. This affects a variation of the Bit Error Rate (BER) in every channel. Fig. 8 illustrates the BER variation vs. operation frequency of the channel for the PCF A, PCF B and PCF D.

For the BER calculation, the Gaussian estimation from the simulator was used, taking as a reference a BER equal to $10^{-12}$ ), in order to secure an appropriate reliability in the link. From Fig. 8 it can be noticed that the operation range of PCF A and PCF $\mathrm{D}$ are similar (taking into account forward error correction techniques on BER values as high as $10^{-3}$ ). Although PCF D offers the lowest BER in most of the range, the length of fiber used was greater than that of the PCF A, hence the accumulated attenuation increases too. This behavior is due to an inferior magnitude of the dispersion parameter of the PCF D. Fiber PCF A showed a lightly superior BER, which can be associated to the greater dispersion slope of the fiber with this configuration.

\section{CONCLUSIONS}

Through this simulation work it was proved that microstructured optical fibers can reduce the length of the conventional dispersion compensating fibers in optical communication links. The purpose of these artefacts are to improve the signal quality, as well as the reach of the link, without requiring major changes over the deployed infrastructure. It was observed the importance of the influence of the microstructure parameters. Specifically, this work evaluated the change in the holes' diameter. It was noticed that varying this parameter in one of the rings alters the dispersion's behavior in all analyzed fibers. Thereby, the results of the PCF compensator show versatility and open potential applications to improve fiber optics telecommunication systems performance. By altering the geometry of these microstructured optical fibers, low dispersion systems can be achieved, as it was shown by the configurations denoted as PCF A, PCF B, and PCF D. These configurations also make possible greater propagation distances as a result of their lower magnitude losses in the C-band. Through varying the parameters of the PCFs, the wavelength at which the fiber compensates the chromatic dispersion can be tuned, making DDCs more versatile. It was found that PCF A obtained a D parameter around $-860[\mathrm{ps} / \mathrm{nm} / \mathrm{km}]$. This result is comparable to that acquired in [24], which was around -612 $[\mathrm{ps} / \mathrm{nm} / \mathrm{km}]$.

\section{REFERENCES}

[1] Govind P. Agrawal, Fiber-Optic Communication Systems, 3rd ed., John Wiley \& Sons, New York (2002).

[2] M. Chen et al., "Chromatic dispersion and PMD monitoring and compensation techniques studies in optical communication systems with single channel speed 40Gbit/s and CSRZ format.," Opt. Express 15(12), 7667-7676 (2007).

[3] D. McGhan et al., "Electronic dispersion compensation," in 2006 Optical Fiber Communication Conference and the National Fiber Optic Engineers Conference, p. 15 pp., IEEE (2006) [doi:10.1109/OFC.2006.215362].

[4] D. Rafique and A. D. Ellis, "Various Nonlinearity Mitigation Techniques Employing Optical and Electronic Approaches," IEEE Photonics Technol. Lett. 23(23), 1838-1840 (2011) [doi:10.1109/LPT.2011.2170193].

[5] S. J. Savory et al., "Electronic compensation of chromatic dispersion using a digital coherent receiver," Opt. Express 15(5), 2120, Optical Society of America (2007) [doi:10.1364/OE.15.002120]

[6] J. Zhao, M. E. McCarthy, and A. D. Ellis, "Electronic dispersion compensation using full optical-field reconstruction in 10Gbit/s OOK based systems," Opt. Express 16(20), 15353, Optical Society of America (2008) [doi:10.1364/OE.16.015353].

[7] N. M. Litchinitser, B. J. Eggleton, and D. B. Patterson, "Fiber Bragg gratings for dispersion compensation in transmission: theoretical model and design criteria for nearly ideal pulse recompression," J. Light. Technol. 15(8), 1303-1313 (1997) [doi:10.1109/50.618327].

[8] I. Riant et al., "Chirped fiber Bragg gratings for WDM chromatic dispersion compensation in multispan 10-Gb/s transmission,” IEEE J. Sel. Top. Quantum Electron. 5(5), 1312-1324 (1999) [doi:10.1109/2944.806756].

[9] S. Spolitis and G. Ivanovs, "Extending the reach of DWDM-PON access network using chromatic dispersion compensation," in 2011 IEEE Swedish Communication Technologies Workshop (Swe-CTW), pp. 29 33, IEEE (2011) [doi:10.1109/Swe-CTW.2011.6082484].

[10] N. Nozhat and N. Granpayeh, "Specialty fibers designed by photonic crystals,” Prog. Electromagn. Res. 99, 225-244, EMW Publishing (2009) [doi:10.2528/PIER09092309].

[11] T. Fujisawa et al., "Chromatic dispersion profile optimization of dualconcentric-core photonic crystal fibers for broadband dispersion compensation," Opt. Express 14(2), 893, Optical Society of America (2006) [doi:10.1364/OPEX.14.000893].

[12] A. Huttunen and P. Törmä, "Optimization of dual-core and microstructure fiber geometries for dispersion compensation and large mode area," Opt. Express 13(2), 627, Optical Society of America (2005) [doi:10.1364/OPEX.13.000627].

[13] N. Janrao and V. Janyani, "Dispersion compensation fiber using square hole PCF," in 2011 International Conference on Communications and Signal Processing, pp. 436-438, IEEE (2011) [doi:10.1109/ICCSP.2011.5739354]

[14] M. Lucki, "Optimization of microstructured fiber for dispersion compensation purposes," in 2011 13th International Conference on Transparent Optical Networks, pp. 1-4, IEEE (2011) [doi:10.1109/ICTON.2011.5971024]. 
[15] X. Zhao et al., "Photonic crystal fiber for dispersion compensation.," Appl. Opt. 47(28), 5190-5196 (2008)

[16] M. A. Islam and M. S. Alam, "Design of a Polarization-Maintaining Equiangular Spiral Photonic Crystal Fiber for Residual Dispersion Compensation Over $\mathrm{E}+\mathrm{S}+\mathrm{C}+\mathrm{L}+\mathrm{U}$ Wavelength Bands," IEEE Photonics Technol. Lett. 24(11), 930-932 (2012) [doi:10.1109/LPT.2012.2190981]

[17] J. C. Knight et al., "Photonic crystals as optical fibres - physics and applications," Opt. Mater. (Amst). 11(2-3), 143-151 (1999) [doi:10.1016/S0925-3467(98)00040-8].

[18] A. Méndez et al., Specialty Optical Fibers Handbook, in Specialty Optical Fibers Handbook, Elsevier (2007) [doi:10.1016/B978-0123694065/50012-6].

[19] T. A. Birks, J. C. Knight, and P. S. J. Russell, "Endlessly single-mode photonic crystal fiber,”Opt. Lett. 22(13), 961, Optical Society of America (1997) [doi:10.1364/OL.22.000961]
[20] A. Ortigosa-Blanch et al., "Highly birefringent photonic crystal fibers," Opt. Lett. 25(18), 1325, Optical Society of America (2000) [doi:10.1364/OL.25.001325].

[21] K. Saitoh et al., "Chromatic dispersion control in photonic crystal fibers: application to ultra-flattened dispersion," Opt. Express 11(8), 843, Optical Society of America (2003) [doi:10.1364/OE.11.000843].

[22] K. Saitoh and M. Koshiba, "Numerical modeling of photonic crystal fibers," J. Light. Technol. 23(11), 3580-3590 (2005) [doi:10.1109/JLT.2005.855855].

[23] D. Chen, M.-L. Vincent Tse, and H.-Y. Tam, "Optical properties of photonic crystal fibers with a fiber core of arrays of subwavelength circular air holes: birefringence and dispersion," Prog. Electromagn. Res. 105, 193-212, EMW Publishing (2010) [doi:10.2528/PIER10042706].

[24] X. Li et al., "Design of a pentagonal photonic crystal fiber with high birefringence and large flattened negative dispersion," Appl. Opt. 54(24), 7350, Optical Society of America (2015) [doi:10.1364/AO.54.007350]. 